\title{
Vejez dependiente, políticas y calidad de vida
}

\author{
M aría Teresa Bazo \\ Universidad del País Vasco / EH U. D epartamento de Sociología \\ Avda. Lehendakari Aguirre, 83. 48015 Bilbao. Spain
}

\section{Resumen}

En el artículo se realiza una exposición y un análisis de las políticas relacionadas con el cuidado de la salud familiar en el contexto actual de cambios demográficos, familiares, económicos, y sociales, que con carácter global afectan a los países de Europa y otras sociedades económicamente desarrolladas, y donde se plantean cambios en los sistemas de bienestar que hagan compatibles el desarrollo económico y el social. A continuación se exponen algunos resultados y al gunas conclusiones de una investigación dirigida y realizada por la autora en el País Vasco, C ataluña y M adrid sobre un tema que hasta recientemente no ha comenzado a investigarse en España: Ios cuidados familiares de salud de las personas ancianas dependientes. El proyecto resulta finalista en la convocatoria de los proyectos de investigación M iquel Angel Terribas de la Fundació "la Caixa". Es seleccionado y financiado por la Fundación Caja de M adrid en una convocatoria nacional, y la investigación recibe el accésit de los Premios Rogeli Duocastella de la Fundació "la Caixa". Se analiza en las tres comunidades autónomas una muestra de un centenar de personas cuidadoras de ancianos y ancianas enfermos crónicos y discapacitados, y personas pertenecientes a la A sociación de Familiares de Enfermos de Alzheimer. Se utiliza la entrevista semiestructurada en profundidad y la entrevista de grupo.

Palabras clave: políticas sociosanitarias, vejez dependiente, calidad de vida, cuidados informales de salud, sistema mixto de cuidados.

\section{Abstract. D ependent old age, policies, quality of life}

An analysis of family health care policies is developed in the article. It is embedded within the change processes that are currently occurring. Those processes are related to demographic, family, economic and social changes, and they affect the European countries as well as other developed societies. Currently, a common concern in those societies is how to deal with the changes that need to be made within the welfare systems, in order to improve both economic and social development. Following such analysis, some research results and conclusions are presented. The research was carried out by the author in the Basque Country, $\mathrm{C}$ atalonia and $\mathrm{M}$ adrid, using qualitative methods. The topic, namely, family heal th care for frail elderly persons, has not been sufficiently studied in Spain. The project and research received several awards from prestigious $C$ atalan institutions, and was partly funded by Fundación Caja de $\mathrm{M}$ adrid. O ne hundred in-depth interviews addressed to careers, and three semi-structured interviews addressed to relatives of Alzheimer' Patients in the three regions were done.

Key words: health and social policies, dependent old age, quality of life, informal health care, welfare mix. 


\begin{aligned} & \multicolumn{2}{c}{ Sumario } \\ & Introducción Resultados \\ & Los retos del envejecimiento Conclusiones \\ & Las políticas sociosanitarias Bibliografía \\ & y el sistema mixto de bienestar \end{aligned}

\section{Introducción}

En las sociedades que envejecen a ritmo creciente, promocionar la calidad de vida en la vejez y en la vejez dependiente es el reto más inmediato de las políticas social es y de los servicios sociosanitarios. El creciente aumento de la esperanza de vida, el descenso sin precedentes históricos de la tasa de natalidad, los cambios en la estructura, en el tamaño, en las formas en la familia, los cambios en el estatus de las mujeres, la reducción creciente de las tasas de actividad laboral entre las personas de cincuenta y cinco y más años, han convertido el envejecimiento de la sociedad ${ }^{1}$ en una cuestión de máximo interés societal.

Son muchas las consecuencias de todos esos procesos, tanto a nivel macrosocial como en las experiencias individuales. Cómo dar sentido a la vida tras una jubilación llegada en muchas ocasiones de forma anticipada e imprevista, cómo hacer frente al mantenimiento de un hogar - en ocasiones con hijos/as dependientes- con una pensión, cómo enfrentarse a la enfermedad crónica y a la dependencia de uno o más miembros ancianos de la familia. Son sólo al gunos temas que necesitan un abordaje teórico y práctico responsable y riguroso. La

1. El término ha sido acuñado y se emplea ampliamente en sociología de la vejez y en gerontología social, siendo aceptado por los expertos en todo el mundo. Se emplea en inglés con el término de ageing society. Incluso se utiliza con frecuencia, aunque a veces entre comillas, el término de graying society, y aunque es más novedoso, ya hablamos de post-retired soci eties, que es un concepto heurístico más que empírico. El concepto «envejecimiento de la sociedad» se refiere a una real idad que sobrepasa la del mero envejecimiento de la estructura demográfica o poblacional, causado por el descenso conjunto de las tasas de mortalidad y de natalidad. Incluso va más allá del fenómeno reciente de la «visibilidad» de las personas de edad. H ace referencia a los cambios profundos que se están produciendo - y se producirán aún más en el próximo futuro - que generan lo que denominamos «una nueva vejez», lo que hace referencia a personas que son más sanas, más educadas, y con más recursos que sus predecesoras, y a las consecuencias que ell o conlleva. Algunas de ellas ponen sobre el tapete los desajustes que se producen en este contexto entre las personas y las estructuras e instituciones, que no se adaptan al envejecimiento de las personas. 0 tras hacen referencia, precisamente, a las políticas públicas, por los retos que les presentan los nuevos dilemas que surgen en las soci edades envejecidas (dilemas o «problemas mal estructurados»). $\mathrm{N}$ o se ven soluciones definitivas en sociedades con economías de crecimiento bajo, estructuras «estables» de población y organización política pluralista. Puede asimismo preguntarse por las políticas de vejez, vistas en términos de equidad vertical a lo largo de las diferentes cohortes, e incluso reflexionar sobre las políticas de bienestar que no proveen necesariamente igualdad horizontal, o justicia a lo largo de todo el ciclo vital, para cada cohorte. Son sólo algunos de los aspectos cuyo desarrollo y reflexión son sugeridos por el concepto «envejecimiento de la sociedad». 
sociedad se encuentra ante nuevos retos para los que necesita instrumentos nuevos. Se requiere un concepto nuevo de solidaridad entre las generaciones y entre los distintos grupos, en un mundo cada vez más complejo, más inseguro, más indeterminado.

La calidad de vida en la vejez tiene que ver con la seguridad económica y con la inclusión social que se asegura por medio de infraestructuras de apoyo y redes sociales. Todo ello promoverá la participación de las personas de edad como miembros activos de la comunidad, una de cuyas funciones puede ser transmitir sus experiencias a las generaciones más jóvenes, al tiempo que comprenden su estilo de vida y los desafíos que les son propios. Todo ello en una sociedad inmersa en procesos que la llevan también a ella a aprender a envejecer.

La calidad de vida en la vejez dependiente implica necesariamente el apoyo social y familiar a las personas que desean continuar viviendo en la comunidad, siendo cuidadas en familia, para que puedan seguir haciéndolo, al tiempo que siguen desarrollándose todas sus potencialidades hasta el último momento. Eso conlleva el apoyo material y afectivo a los familiares que, con distintos grados de implicación, participan en la acción de cuidar. Políticas que tengan presente la dimensión femenina de los cuidados de salud, para que no contribuyan a seguir reforzando el rol dependiente de las mujeres cuidadoras.

En el artículo realizaré un análisis de las políticas relacionadas con el cuidado de salud familiar en el contexto actual de cambios demográficos, familiares, económicos y sociales que, con carácter global, afectan a los países de Europa y otras sociedades económicamente desarrolladas, y donde se plantean cambios en los sistemas de bienestar que hagan compatible el desarrollo económico y el social. Expondré finalmente al gunos resultados y al gunas conclusiones de una investigación que he dirigido y realizado en el País Vasco, C ataluña y M adrid².

\section{Los retos del envejecimiento}

Las personas en la actualidad están al canzando cada vez edades más avanzadas en mejor estado de salud. Pero precisamente el hecho de que se viva más conlleva mayores probabilidades de tener enfermedades crónicas e invalidantes. Aumenta la invalidez, la discapacidad y la minusvalía. A pesar de las amenazas al Estado de bienestar, el análisis de costos no puede realizarse únicamente

2. El proyecto resulta finalista en la Convocatoria de los proyectos de investigación M iquel Angel Terribas de la Fundació "la Caixa". Es seleccionado y financiado por la Fundación C aja de $M$ adrid en una convocatoria nacional, y la investigación recibe el accésit de los Premios Rogeli D uocastella de la Fundació "la C aixa". En la investigación se analiza en las tres comunidades autónomas una muestra de un centenar de personas cuidadoras de ancianos/as enfermos crónicos y discapacitados, y personas pertenecientes a la Asociación de Familiares de Enfermos de Alzheimer. Se utiliza la entrevista semiestructurada en profundidad y la entrevista de grupo. Participa en el estudio Carmen Domínguez-Alcón, de la Universitat de Barcelona. 
desde una perspectiva de mercado. Se considera que el envejecimiento de la población amenaza el sistema de salud. Se sabe que las personas mayores consumen más servicios sanitarios y más medicamentos que otros grupos de edad. Pero las pautas de consumo no son homogéneas para todo el grupo de personas de sesenta y cinco y más años. Se concentra en las edades más avanzadas y en el período previo a la muerte (Borgatta y M ontgomery, 1987: 7-22). O curre también en la actualidad que el uso cada vez más alto de servicios por parte de las personas mayores se combina con un incremento alto del costo de los servicios médicos.

Respecto al futuro es preciso analizar ciertos factores. La relación de la esperanza de vida, longevidad, y prevalencia y duración de las enfermedades crónicas definirán el nivel de dependencia entre las personas ancianas, lo que a su vez influirá en el sistema sanitario.

El impacto en el sistema de salud del notable volumen previsto de personas ancianas será consecuencia de las condiciones de vida de la población y del alcance de la dependencia. Las consecuencias de la mejora de las condiciones de la población anciana puede influir en los cambios de las políticas. Con respecto a la Seguridad Social, se espera que la edad de la jubilación se retrase a los setenta años. D e hecho ya ocurre para los profesores y profesoras de universidad. Cuando a principios de siglo se establece la edad de jubilación a los sesenta y cinco años pocas personas llegaban a jubiladas, y sólo una pequeña proporción vivía el tiempo suficiente para disfrutarla. Las condiciones generales de vida y trabajo están cambiando aceleradamente.

Si no resulta descabellado pensar en prolongar la edad de jubilación, es que el deterioro severo de la salud llega más tarde, que los costos se están posponiendo. Si no se produce ningún otro cambio puede esperarse sencillamente que el volumen de cuidados de salud que se requería anteriormente se experimentará por las personas a una edad más tardía, pero las personas en un momento dado necesitarán los cuidados. Se observa la importancia para la política de salud de la investigación e información en los cambios en los niveles de salud en la estructura de edades cambiante. Interesa sobremanera investigar sobre el comienzo y la duración de las enfermedades crónicas.

En cuanto al índice de dependencia, se prevé que aumente y que cambie su estructura, recayendo el mayor peso sobre las personas mayores, al tiempo que disminuye el de las más jóvenes. Pero si se prolonga la edad de jubilación la definición del concepto y los resultados de la relación variarán y puede que en las próximas décadas el cambio no resulte significativo proporcionalmente. Sin embargo, no se alterará el hecho del incremento potencial del período de dependencia en las personas ancianas previo a la muerte.

Una preocupación de los gobiernos en la actual coyuntura es cómo compaginar las políticas educativa, de salud y laboral entre sí, y con la económica. D esde los gobiernos se invita a compartir las responsabilidades entre el Estado, la familia y el individuo; entre el sector público y el privado. En cuanto al gasto público, se dice, deduciéndolo así del incremento habido en las últimas décadas, que continuará aumentando en el futuro. Pero una parte 
considerable de ese incremento se debe a la maduración de los sistemas de pensiones y de salud.

Por otro lado, en el notable aumento del gasto sanitario en los últimos años en los países de la O CD E la proporción estimada de ese incremento imputable a factores demográficos es de alrededor del 13\% (O CDE, 1987). Además, el mayor gasto es realizado sólo por una pequeña parte, que son las personas más ancianas (O ry y Bond, 1989). D e considerable interés resulta destacar así mismo otro aspecto, y es el desconocimiento en cuanto a los modelos de morbilidad respecto al futuro. Existen dos hipótesis fundamental es y contradictorias. U na hace referencia al incremento considerable del gasto sanitario debido al aumento de la población anciana y sobre todo de la de más edad, dado que es el grupo con mayores probabilidades de experimentar invalidez y enfermedades crónicas. La otra se refiere a la consideración de la mejora de la salud de las personas, que envejecen cada vez más, pero en mejores condiciones. Se estima así que los factores sociales que han influido en esa situación (vivir más y en mejor estado de salud) pueden influir también en la reducción de las tasas de dependencia retrasán dola hasta el último tramo de la vida previo a la muerte. Se argumenta que si hace cincuenta años se hubiesen realizado proyecciones sobre dichas tasas para el final del siglo, la estimación habría sido notablemente superior (M ontgomery y Borgatta, 1987: 235-252). En este sentido, resulta pertinente argumentar en favor de políticas económicas, fiscales, laborales y social es que promuevan el bienestar a lo largo de toda la vida, y por tanto de la prevención, que, dicho sea de paso, conlle vará ahorro económico.

Es importante investigar en este campo y analizar la longevidad. El número de personas centenarias aumenta en el mundo. También el interés por su estudio. D e las investigaciones existentes se desprende que la mayor parte de las personas longevas viven en un buen estado de salud relativo y una buena parte de ellas son total o casi totalmente autónomas para realizar las actividades de la vida diaria (Fraiz Calvo, 1993; Sánchez-O stiz, 1997). D iríase que cuando las personas logran al canzar una determinada edad, en muchas de ellas aumenta la probabilidad de vivir en buenas condiciones de salud.

Se observa vigor, vitalidad y buen estado mental en muchas personas de edad avanzada e incluso centenarias. Son aspectos que es necesario investigar para conocer mejor las potencialidades humanas y poder hacer mejores previsiones sobre los futuros model os de morbilidad (Bazo, 1992a).

Es cierto que entre las personas mayores pueden observarse en distinto grado y extensión algunos problemas que les afectan precisamente por su condición de jubiladas y ancianas. El principal problema de las personas ancianas desde el punto de vista económico es la pobreza (Walker, 1991a). Las personas después de la jubilación tienen más probabilidades de encontrarse en el umbral de la pobreza o por debajo del mismo, que antes de la jubilación. La pobreza en la vejez persiste a pesar de que el reconocimiento social del problema viene de largo. En España, según el Informe Foessa, el $33 \%$ de las personas ancianas son pobres, y alrededor del $43 \%$ de todos los 
hogares que se encuentran por debajo del umbral de pobreza, el cabeza de familia es una persona de sesenta y cinco o más años (Rodríguez C abrero, 1994: 1.430). Las prestaciones sociales fallan a la hora de hacer frente a las necesidades de quienes las reciben, Io que conduce a muchas personas a llevar una vida de lucha por la existencia. La pobreza conduce a la carencia de los bienes básicos, con las consecuencias que ello tiene para la calidad de vida de las personas.

\section{Las políticas sociosanitarias y el sistema mixto de bienestar}

El otro gran tema de debate en la elaboración de las políticas y provisión de los servicios sanitarios y sociales es el cuidado de las personas ancianas y, subsecuentemente, el reparto de las responsabilidades. Interesa por tanto analizar la forma en que políticas y servicios públicos se relacionan e interactúan con las actividades privadas.

En la actual coyuntura donde se cuestiona la continuidad, tal como ha llegado a desarrollarse, del Estado de bienestar, se entiende que la cuestión de la provisión de los cuidados a las personas más ancianas - que son las que proporcionalmente más aumentan- es una cuestión de vital importancia. Se hace un Ilamamiento al sector informal, pero se teme que los cambios familiares operados neutralicen el potencial de cuidados que puede proporcionar en el próximo futuro. Es por lo que se apela «ansiosamente» (Abel, 1989) a la familia y a los amigos para que asuman responsabilidades adicionales en el cuidado de las personas ancianas.

Se considera que en la práctica existe una interdependencia entre los sectores informal y formal, ya que el sector formal sería incapaz de asumir los cuidados de todas las personas dependientes, y porque el sector informal es en parte función de la oferta y distribución de los servicios sociales y de las políticas económicas y sociales del Estado (Q ureshi y Walker, 1991). Sin embargo, en España la escasez de los servicios de cuidado comunitario provistos por el sector público y la amplitud, por el contrario, de los servicios informal es provistos por la familia cuestionan tal interdependencia. Es decir, que el sector formal aparece en la mayor parte de las comunidades autónomas como un mero apéndice que de ser suprimido apenas se notarían los efectos. Y no lo serían por una doble razón: en primer lugar por su débil impacto social, en segundo - y sólo en parte relacionado con lo anterior - por la escasa demanda, en relación con las necesidades, por parte de las familias. U na causa que influye de manera considerable en que las familias no demanden más servicios según sus necesi dades reales, es el sentimiento general izado entre la población de la asunción del cuidado de las personas mayores de la familia como parte de los deberes familiares. Son actitudes que incluso la generación de jóvenes ha interiorizado (Bazo, 1994a).

El cuidado familiar se ha basado en el tiempo y el trabajo de las mujeres, que se supone es libre en términos económicos. Actualmente se entiende que la disminución del tamaño familiar, la incorporación de las mujeres al mundo 
laboral, sus niveles más altos de instrucción, el aumento de los divorcios, los nuevos matrimonios y los cambios que todo ello conlleva en los modelos tradicionales de familia en las sociedades denominadas occidentales, pone en peligro la continuidad de dichos cuidados.

$\mathrm{H}$ acer política social significa tomar unas decisiones que se realizan en un contexto económico, cultural e ideológico. La concepción de la familia en el sentido tradicional puede conducir a invalidar las políticas social es. Las diferentes situaciones de las sociedades en cuanto a sus características demográficas que afectan al tamaño y a la estructura de la familia, las tasas de participación femenina en el mundo del trabajo y las variaciones en la familia extensa son aspectos a tener en cuenta.

Las políticas y los servicios sociales se elaboran teniendo en cuenta una concepción determinada de la familia y de la comunidad. La familia se ha entendido compartiendo responsabilidades entre sus miembros y ofreciéndose mutuamente contraprestaciones, como algo propio de las «obligaciones familiares». Todavía hoy la pal abra obligación se escucha de las personas que cuidan familiares enfermos crónicos.

Por otro lado, la concepción de la familia se basa también en una estructura en la que el varón es el proveedor de los ingresos y la mujer la proveedora de los cuidados y el servicio. La diferenciación de roles en la familia lleva a las mujeres a especializarse como amas de casa y madres. Se considera que su tiempo empleado en el desempeño de tal es roles es libre en términos de disponibilidad y costo (Allan, 1990: 17). Cuando se crea la Seguridad Social, aunque se tiene en cuenta que algunas mujeres casadas participan en el mercado de trabajo, la idea que predomina es la del varón que gana el sustento y mantiene al resto de los miembros dependientes de la familia (esposa e hijos principalmente). Se considera que la creación de los estados de bienestar se basó en el trabajo no pagado de las mujeres en el hogar, y en el pagado pero con salarios bajos de las mujeres en las instituciones de bienestar. D el mismo modo, la estructura de prestaciones reflejaba el estatus dependiente de las mujeres y los deberes domésticos como su prioridad, mientras se entendía que la responsabilidad primordial de los varones se encontraba en el mercado de trabajo. Sigue sucediendo que las políticas sociales actuales que ponen el énfasis en el cuidado comunitario tienden a reforzar la ideología del familismo al asumir que la familia es el lugar idóneo para el cuidado de las personas ancianas enfermas, y que las muje res son los familiares más apropiados para asumir esa tarea (Walker, 1991b).

En cuanto al servicio público de salud, se supone que provee de los cuidados necesarios a las personas enfermas. Sin embargo, en ciertas sociedades se combina el servicio público con el privado. Pero la mayor parte del cuidado que necesitan las personas enfermas es recibido fuera de los servicios formales de salud, y es proporcionado por la familia en primer lugar, y también - en ciertas sociedades sobre todo- por amigos y vecinos y asociaciones altruistas, especial mente en lo que se refiere al cuidado y a la atención diarios. Esa relación de dependencia entre el Estado y las familias es un aspecto fundamental a tener en cuenta, sobre todo por las consecuencias que tiene para las muje- 
res, cuya contribución es solicitada de forma desproporcionada. En Gran Bretaña la proporción de ancianos y ancianas con niveles altos de dependencia que son cuidados por su familia es más de tres veces la proporción de los que se encuentran en todas las instituciones de salud y servicios sociales juntas. Incluso el grupo que sufre al gún tipo de demencia, que es el grupo cuyo cuidado causa las tensiones más graves entre las personas que les atienden, las cua tro quintas partes son cuidadas en el seno familiar. Es algo parecido a los datos conocidos sobre C anadá (Walker, 1991b).

Aunque en el País Vasco, N avarra o Cataluña los servicios social es pueden tener un desarrollo algo más amplio que en otras comunidades autónomas, lo cierto es que en España la importancia de la familia como cuidadora es abrumadoramente superior a la que puede tener en otras sociedades con sistemas de protección social más extensa e intensivamente desarrollados. Se observa desde una perspectiva internacional que existe una relación inversamente proporcional entre la utilización de las fuentes de cuidado formal einformal. A mayor desarrollo de las funciones protectoras del Estado, menor (y distinta) aportación familiar, y viceversa. Sin embargo, en todas las sociedades la familia sigue siendo fuente fundamental de cuidados (Tennestedt y M cKinlay, 1989; J amieson ellisley, 1993; M ontgomery, H atch y otros, 1987).

Se entiende que en las dos o tres últimas décadas en el plano internacional se está produciendo un «descubrimiento de los cuidadores» aunque hayan existido siempre (H unter y M acpherson, 1993: 67). Por otra parte, el rol de cuidadoras de las mujeres es visto como un rol que toman de por vida como la promesa del matrimonio «hasta que la muerte nos separe» (Peace, 1991). El cuidado de las personas ancianas, y entre ellas las mujeres son mayoría, sigue corriendo principalmente por cuenta de las mujeres de la familia.

Últimamente, y debido a la presión sobre los presupuestos, se insiste en el apoyo informal (sobre todo el familiar) y se pretende reforzarlo bajo el supuesto de su nulo o menor coste económico. Al mismo tiempo, ciertos estudios muestran la adhesión de las mujeres a su rol de cuidadoras y la creciente implicación en los cuidados de sus antepasados ancianos a medida que ellas mismas envejecen. Los cuidados de los familiares enfermos crónicos suponen costos de carácter emocional, de salud y económicos para quienes los proveen, que en ocasiones son considerables según ciertas investigaciones (Peace, 1991: 69; Bazo y D omínguez, 1996).

En Europa se observan orientaciones distintas para las políticas, que van desde el intento de sustituir la atención comunitaria por los servicios informales, pasando por quienes pretenden completar y apoyar los servicios informales, hasta quienes proveen a las personas ancianas de los cuidados comunitarios necesarios sin complemento de los informales (Jamieson elllsey, 1993). En Europa, en distintos países, se conceden remuneraciones a las personas que prestan servicios asistenciales informales, tanto a las personas ancianas o enfermas como a los hijos y/o hijas (Evers y Leichsenring, 1994). 0 tra cuestión a analizar es si las políticas sociales deben tener en cuenta las diferencias entre los distintos modelos de relación familiar, las divisiones por géne- 
ro en el cuidado familiar y las diferencias estructurales entre familias según la clase social.

Se observa que en la mayor parte de las sociedades la familia no es sólo la principal fuente provisora de cuidados (función que ejerce en la misma medida después de crearse los sistemas de seguridad social y de servicios sociales), sino también la instancia mediadora más importante entre las personas ancianas y las burocracias (Gibson, 1992). Q ue la familia pueda fallar en sus funciones cuidadoras, sobre todo por razones demográficas y económicas, podría suponer en los países desarrollados la peor crisis económica para los sistemas de salud y de servicios sociales. D esde esa misma perspectiva se entiende que la próxima crisis del Estado de bienestar no será la prevista para el año 2010, cuando las generaciones más numerosas Ileguen a la jubilación, ni tampoco la que provenga del crecimiento de la demanda debido al aumento de las personas mayores. La crisis será una crisis en la provisión de cuidados, debido a la disminución notable de la cantidad de tiempo dedicado al trabajo no pagado por parte de las mujeres, dada su creciente participación en el mercado de trabajo (M yles, 1991).

En cuanto a la economía mixta del bienestar, en general siempre ha existido ese sistema mixto dentro del Estado de bienestar. H a coexistido la medicina privada y la pública, y lo mismo en la educación. En cuanto a la política de vivienda, ésta ha sido estimulada por los estados, pero coexistiendo con el sistema cooperativo y la actividad privada. La cuestión actualmente es, y dadas las presiones que en el plano internacional se ejercen en cuanto a las restricciones en el gasto social, en qué medida el sector informal y el mercado podrán hacer frente a ese reparto de responsabilidad.

Existe una serie de ideas acerca de las bondades de la economía mixta de bienestar. Suele decirse que el sector privado es más barato que el público, y que mantiene una mejor relación costo-eficacia. Sin embargo, parece que no es necesariamente siempre así (Walker, 1984: 38-39). O tra idea es que la obtención de fondos por parte de las asociaciones de voluntariado - en los países donde existe una tradición altruista - hace más fácil recaudar los recursos. Sin embargo, como es el caso de Gran Bretaña, se observan limitaciones en el presente tanto en los ingresos, que se han visto reducidos por la recesión económica, como en el número de personas que se siguen incorporando a las asociaciones (M ayo, 1994: 30). En España las asociaciones de voluntariado se nutren de fondos públicos, aunque la mayor parte de las veces en cantidades simbólicas, así como de las cuotas modestas de sus asociados/as. Pero también en otros países ocurre al go parecido. Puede ocurrir igualmente que aumentando incluso en ciertos casos la aportación de los fondos públicos en asociaciones de voluntariado, sin embargo, cada vez tenga el Estado menor responsabilidad en las actividades realizadas.

No existe tradición en España de que las empresas aporten cantidades a obras benéficas como en el mundo anglosajón. Las fundaciones que se crean dedican en general sus fondos primordialmente a obras culturales (digámoslo así en sentido amplio) y no tanto a obras sociales. Incluso entre las cajas de aho- 
rros, que constituyeron en décadas pasadas un apoyo importante en el sistema de bienestar, sosteniendo escuelas, institutos, servicios específicos de salud, residencias de ancianos o guarderías, se observa un cierto abandono progresivo de esas funciones. Con los cambios económicos que están conduciendo al empobrecimiento de ciertos sectores sociales y a la falta de atención de las necesidades de al gunos grupos específicos, podría ser el momento para las cajas de volver a sus orígenes, iniciando y promoviendo actividades que vuelven a ser de necesidad social.

O tro aspecto que también se ha idealizado en cuanto a la actividad de los sectores no públicos, es el relativo a la posibilidad de una mayor elección de los usuarios dentro de una economía mixta de bienestar. La realidad es que dicha elección no existe para todas las personas. Aquéllas que pueden acceder a los servicios privados son sólo las que pueden pagarlos. En España lo que sucede es que las autoridades de servicios sociales conciertan unas plazas o servicios con el sector privado. Puede ocurrir, sin embargo, que, debido a las restricciones económicas, por ejemplo existan plazas en residencias sin cubrirse, al tiempo que las listas de espera aumentan progresivamente. Cuando se dice que una economía mixta de bienestar trae consigo una mayor posibilidad de elección se olvida que las personas ancianas en este caso, que están en situación de fragilidad, no se encuentran en las condiciones más adecuadas para ponerse a buscar entre las diversas alternativas que les ofrece el mercado abierto.

0 tra de las supuestas bondades del sistema mixto es la relacionada con la presunta y mejor participación de las personas en la toma de decisiones dentro del sector del voluntariado. Sin embargo eso no es necesariamente así siempre, dado que en muchas ocasiones las decisiones sobre la asignación de los recursos se toman en otras instancias, sean públicas o privadas.

Sin embargo, desde ciertas perspectivas ( $M$ ayo, 1994: 40-42) se entiende que los sectores no estatales tienen importantes funciones que cumplir, como - en los casos de ciertas organizaciones de voluntariado- la de ejercer una vigilancia respecto a que se eviten ciertos efectos no deseados que acompañan en ocasiones al funcionamiento de la burocracia, que es sin embargo - en el sentido weberiano- el desarrollo de la racionalidad en las organizaciones complejas públicas o privadas. 0 tra cuestión que puede ser objeto de esa atención es la promoción de la participación democrática como forma de presionar en el sector público. Por otro lado, parece que cualquier intento de sustituir de forma masiva los servicios públicos por los privados, conduciría a una mayor desigualdad. Además, tampoco se ve como una alternativa realista, y los fondos públicos serán necesarios siempre. Parece así mismo que el sector voluntario debe seguir ejerciendo una función complementaria y nunca sustitutoria.

En cuanto al futuro desarrollo en Europa de unas estructuras de bienestar comunes, puede decirse que de momento no existe una política social en la Unión Europea. Tanto el Acta Europea de 1986, como el tratado de M aastricht de 1991, como la reforma fallida en el tratado de Amsterdam (1997) conducen a Europa a una comunidad económica, política, y en cierto modo social, pero no se entrevé todavía un Estado de bienestar europeo, temiéndose que si 
permanece la ciudadanía social sólo a nivel nacional se produzca con el tiempo una lenta erosión de la misma. Conseguir una Europa social o ciudadanía social europea es más complejo que lograr un mercado común. N o perseguir esa meta es arriesgarse a afrontar graves riesgos para la consolidación del proyecto europeo. Se entiende que «sin un Estado de bienestar europeo, a largo plazo, los sistemas de bienestar nacionales se atrofiarán: sus bases económicas y legitimadoras podrían erosionarse progresivamente conforme se acometa y desarrolle el M ercado C omún» (Liebfried, 1993: 139).

Sí comparten los diversos países, sin embargo, una problemática común, como puede ser el desempleo y los problemas sociales que surgen derivados del mismo, al tiempo que se producen otros cambios demográficos, culturales y sociales. Comparten también una misma preocupación por el cuidado y la atención de las personas con necesidades específicas, como es el caso de las ancianas en situación de dependencia. Les preocupa cómo hacer frente al incremento que parece inexorable al menos a medio plazo, del gasto social en pensiones, salud y servicios sociales. Se teme que la familia falle en sus tradicional es funciones cuidadoras, tanto porque las necesidades económicas, así como nuevos valores, conducen a las mujeres - tradicionales cuidadoras- a participar más intensa mente en el mercado de trabajo, como por los cambios ocurridos en la estructura y tamaño de las familias (Walker y Warren, 1993). Los problemas de las propias familias cuidadoras, la responsabilidad desmedida cargada cultural y estructuralmente sobre las mujeres, son los mismos en los diversos países. Lo que sucede es que esos problemas se perciben de forma distinta según la situación económica, tradición en los sistemas de bienestar, y por tanto en las expectativas que se generan, al margen de la comparación cuantitativa entre países. En Europa, sin embargo, sigue predominando la idea de que las personas mayores deben ser cuidadas y atendidas en la comunidad (Walker, 1991b).

El futuro de las personas ancianas en Europa, cada vez más numerosas y saludables, pero que por ser numerosas y más ancianas aumentará probablemente en cifras absolutas el número de quienes necesitarán más cuidados, es un asunto que compete a las sociedades, a las familias y a los individuos, a los esta dos nacionales y a la U nión Europea. La ciudadanía europea se basará sobre todo en los derechos reconocidos de todas las personas pertenecientes a cualesquiera grupos sociales, independientemente de su edad, género o condición social . O no existirá una Europa de los ciudadanos y de las ciudadanas.

\section{Resultados}

Puede decirse que las familias se enfrentan en soledad a la situación de cuidar a una persona anciana que sufre una enfermedad inval idante. Siendo Euskadi y $C$ atalunya dos comunidades que cuentan con mejores sistemas sanitarios y sociales que otras, sin embargo, los servicios comunitarios ofertados a la población anciana que los necesita están claramente por debajo de las necesidades.

En la presente investigación se observa que las primeras reacciones de los familiares entrevistados ante los acontecimientos que provocan una enferme- 
dad invalidante (como un accidente cerebrovascular) suelen ser de dolor y estupor. N o obstante, en el primer momento se confía en la posibilidad de una mejoría, si no de una recuperación. Al final suele aceptarse la dependencia de la persona anciana, pero en al gún caso detectado ni siquiera se trata de resignación. Se hace frente al hecho con una profunda frustración.

El trato y la relación previos a la enfermedad determinan, junto con otras variables (rasgos de personalidad, religiosidad), la reacción ante el hecho, y también el trato con la persona enferma y el ambiente familiar posteriores, así como los niveles de estrés, salud y sentimientos de bien estar/mal estar de la persona cuidadora. En las personas de edades avanzadas son sobre todo las hijas, la mayor parte casadas, quienes se ocupan de un padre o de una madre discapacitado. Si hay una hija soltera suele ser ella, aunque trabaje, la que se ocupa del enfermo o de la enferma. En tercer lugar, será una nuera - o variaslas que se hagan cargo.

Se manifiesta con frecuencia la dureza de la situación a la que al guien denomina «tragedia». A veces, después de haber muerto la persona cuidada se reconoce incluso una cierta inconsciencia en el pasado. Es decir, que a posteriori se ve que la situación pasada no fue valorada por ella en ese momento con toda la carga de trabajo, esfuerzo y sacrificio que suponía. Pero son numerosas las declaraciones en el sentido de que la experiencia ha supuesto también una compensación humana y afectiva. A pesar de las dificultades, se observa que la familia siente en general la necesidad de cuidar de sus familiares enfermos. Bien es cierto que la mayor parte de las personas entrevistadas manifiestan que se cuida a los enfermos crónicos en casa porque no existen centros adecuados para atenderles, o porque son muy caros.

La constatación de la mayor incorporación al mundo laboral y profesional de la mujer vuelve evidente la necesidad de otros recursos para atender necesidades que todavía son resueltas en exclusiva por las mujeres en la mayor parte de las ocasiones. Entre las personas entrevistadas en al gún caso se entiende que en el futuro las cosas cambiarán debido a los valores más hedonistas de las generaciones jóvenes.

$M$ anifiestan en general un asentimiento en cuanto a la necesidad de la existencia de plazas en residencias asistidas debido al esfuerzo que supone para las familias atender - durante años en ocasiones- a personas ancianas discapacitadas. Pero, al mismo tiempo, se cree que nunca estarían tan bien cuidadas como en familia. Parece observarse de forma implícita una contraposición entre amor y técnica, una valoración mayor del afecto familiar que del conocimiento y la atención profesionalizados.

Son numerosos los casos en los que se habla de obligación en la atención familiar, aunque en otros se descarta abiertamente. D ice una mujer que cuida de su madre: «C reo que mi obligación es atender a mi madre lo mejor posible y hacerle feliz. El verla contenta me llena de ilusión». O tra cuidadora que colabora en días alternos en el cuidado de su suegro con otras cuñadas, debido a que los hijos no quieren tener una tercera persona pagada, comenta: «N o recibo ninguna compensación económica por parte de mi suegro. Le cuido 
porque es un familiar mío y es una obligación para mí. Poca gente cuida a un enfermo por voluntad propia».

En al gunos casos se observa la presión familiar, o conyugal, para hacer frente a una situación que resulta costosa desde el punto de vista personal. Los val ores acerca de la supuesta tendencia natural en las mujeres para el cuidado, así como la asunción de que es algo asociado a su rol, lleva a esperar de ellas su aceptación - a veces forzada - de unas obligaciones que por sí mismas ellas no hubieran deseado asumir. Se habla también de deber, refiriéndose a correspondencia. Es decir, ha llegado el momento de devolver, de compensar al padre o madre enfermo o enferma, por sus cuidados, amor y desvelos anteriores. $\mathrm{H} \mathrm{a}$ llegado el momento de saldar la deuda de la vida. En este sentido, son diversas las investigaciones donde aparece la idea de compensación o devolución (Shulz, 1990). L ewis y M eredith (1988: 28) encuentran que los sentimientos de afecto y obligación son como las dos caras de una misma moneda. Por su parte, U ngerson (1987: 92) distingue entre las motivaciones para el cuidado de los varones, expresadas en términos de amor y las de las mujeres en términos de deber.

Pero son muchas las dificultades señaladas que ponen en peligro en bastantes casos la continuidad de los cuidados familiares. Es habitual la alteración de la vida familiar e incluso el surgimiento de conflictos sobre todo entre la pareja, aunque aparece más comúnmente señalado el apoyo del cónyuge que el conflicto con él. Son numerosos los casos en los que se habla de ruptura de costumbres tales como ir la familia de vacaciones, no poder salir en pareja, o con los niños, o al menos en la medida en que era habitual antes. La situación - que puede ser más o menos prolongada - de cuidar a una persona anciana discapacitada, afecta y altera la vida familiar en sus rutinas, y a las personas cuidadoras en sus posibilidades de elección de actividades y del propio autocuidado, condicionando su vida en ocasiones de forma notable. Influye también en la relación convivencial. En bastantes casos se comenta el apoyo de la pareja y su comprensión de la situación.

En otros casos, por el contrario, la situación es más difícil: «Entre nosotros ha habido una gran ruptura. Los que se han visto más influidos han sido mis hijas y mi marido, sobre todo él se siente totalmente desplazado. V iene de trabajar y no tenemos tiempo de hablar entre nosotros, no podemos salir ni podemos quedar con otros matrimonios. Esto le ha afectado mucho. N uestras relaciones en muchos momentos son tensas. M e debato entre dos polos opuestos. Por una parte ella es mi madre, y por otra él es mi marido. En algunos momentos siento que me tengo que decidir por alguno de ellos, pero no sé qué hacer. La convivencia entre nosotros es muy difícil y le debo agradecer que sea tan comprensivo». Un apoyo exterior, al menos para los fines de semana, podría suponer un alivio a las tensiones generadas por el cuidado constante de una persona anciana enferma, una mejora para la calidad de vida de la persona cuidadora, pero también de su familia y de la propia persona cuidada.

Las experiencias de las personas entrevistadas y sus familias son similares a las encontradas en otras sociedades con más y mejores servicios sociales. Ciertas 
investigaciones (Policy Studies Institute, 1982) destacan las mismas situaciones: tensiones familiares, ruptura en la vida familiar habitual, el triángulo que se forma en el que la esposa debe hacer frente a las responsabilidades y requerimientos tanto del marido como del padre o madre enfermos. En la investigación británica citada las mujeres entrevistadas y sus esposos, manifestaban como principales consecuencias de la acción de cuidar de un anciano o de una anciana dependiente, la incidencia negativa en las relaciones de pareja y/o con los hijos e hijas. Las mujeres lamentaban especialmente - igual que ocurre en la presente investigación en el País Vasco, C atalunya y $\mathrm{M}$ adrid- no poder dedicar más tiempo a sus hijos y/o hijas, ni compartir con ellos juegos y otras actividades. Por otro lado, el tamaño reducido de las viviendas y la falta de privacidad que conlleva contribuye también a alterar la convivencia familiar y se generan tensiones. La propia configuración de las viviendas no facilita la atención de la persona discapacitada.

Un aspecto importante es los cambios experimentados por la propia persona cuidadora en el proceso de cuidar. Se hace referencia a deterioros en la salud física y psíquica - son varios los casos en que se habla de depresión- y a alteraciones del carácter. La influencia de la acción de cuidar en el carácter, ánimos y salud de la persona cuidadora es un aspecto a resaltar. Son raros los casos en los que claramente se señala que no ha existido una influencia negativa en este sentido. En un caso, incluso, encuentra más agradable la dedicación al cuidado de la madre que el de puericultora en una guardería.

Son numerosas, por el contrario, las situaciones opuestas. Se trate de los padres biológicos o políticos, se manifiesta que el impacto de la situación en la persona cuidadora es duro y con consecuencias negativas. Influye el ver que no existen esperanzas de recuperación o mejora sustancial del enfermo o enferma. Una explica con dramatismo la situación: «M i vida ha cambiado mucho, es más amarga, mucho más agria. M e ha cambiado el carácter, porque mi madre ¡no me conoce! ¡N o sabe quién soy! Esto ocurre día tras día durante tres años [... ] Cada dos horas tengo que llevarle al servicio, así está mi espalda como está». Es interesante notar que en nuestra sociedad la fal ta de medios técnicos y humanos incrementa las dificultades y consecuencias negativas del cuidado de una persona discapacitada. N uestra sociedad, en comparación con otras del contexto europeo, no dispone de recursos suficientes para ayudar a las familias a ocuparse de las personas con problemas de dependencia.

La influencia en el estado de ánimo es considerable. La que ha perdido el empleo comenta: «D esde entonces no se puede decir que todo haya sido igual. Estar todo el día con ella no supone un trabajo físico el evado, sino verte a ti misma cada día peor. $\mathrm{H}$ ay días en los cual es creo que estoy peor que ella, por lo menos psicológicamente, aunque luego hago todo lo que está en mi mano para que ella no se dé cuenta [... ] M e siento cansada, sola, los problemas los vemos nosotras que convivimos con el enfermo, pero me suele dar la impresión de que nadie más me comprende. M i estado de ánimo podría resumirlo con una palabra: depresión». En otras investigaciones (Policy Sutudies Institute, 1982) se aprecia también la situación de impotencia, y de no poder seguir 
adelante, de muchas mujeres cuidadoras, así como el aislamiento social en que se encuentran, debido a la pérdida paulatina del trato con las amistades y personas con las que se relacionaba antes de involucrarse en la acción del cuidado.

La sobrecarga física y emocional que supone para las personas cuidadoras - en su mayoría mujeres- hacer frente a la situación sin disponer de suficiente formación, recursos económicos y materiales, apoyo de profesionales y de los servicios sanitarios y social es, tiene consecuencias negativas en ellas, en su salud física y mental. Pero hay - según se observa en ciertos casos- un factor añadido cuando la persona cuidada es un padre o una madre político, sobre todo cuando la relación anterior no era positiva. También se observa en al gún caso en la presente investigación que las personas que cuidan de un familiar enfermo pueden expresar un rechazo al cuidado realizado por personas extrañas a la familia, incluso siendo profesionales. Es algo que ya detectaron Lewis y M eredith (1988).

$\mathrm{H}$ ay un aspecto que resulta interesante analizar. Se refiere a los procesos de negociación que se producen a la hora de atender a una persona enferma y los procesos de asignación de un miembro de la familia como cuidador o cuidadora. Los procesos pueden comenzar, como señala Ungerson (1987: 5) entre los hijos y/o hijas y el padre que es el cuidador principal - 0 único- y que comienza a tener problemas para poder proseguir con su tarea. Pero también se producen entre las hijas y las otras partes interesadas en sus servicios: sus maridos e hijos y/o hijas. Entiende esa autora que «el proceso de llegar a ser cuidadora, y la forma en que la edad, el sexo, y el estatus marital influyen son construcciones sociales» (p. 57). Lo que se observa en la presente investigación es sobre todo una negociación entre los diversos hermanos y hermanas entre sí. A veces el resultado parece que es, simplemente, que al guien acaba aceptando - y asumiendo para sí- la situación.

A veces llegan a acuerdos sobre la forma de compartir el cuidado, bien acudiendo al hogar del enfermo o de la enferma a una hija o nuera durante un período determinado (puede ser una semana), bien teniendo al anciano 0 anciana en la casa propia durante ciertos meses al año. 0 tras veces, puede que un hermano (más bien una hermana) cuide del enfermo o de la enferma durante todo el año y que en verano durante dos meses se haga cargo otro hermano 0 hermana. En alguna ocasión, los hermanos o hermanas que no cuidan habitualmente, lo hacen los fines de semana para permitir el descanso de la familia cuidadora principal.

Pero esos procesos de negociación - que aparecen con más claridad en la entrevista de grupo- quedan más diluidos en las entrevistas personales. Aparecen sin embargo con mayor claridad - aunque sean minoritarios- los procesos de asignación. Claro está que en muchos casos unos procesos y otros se solaparán y será difícil deslindar uno de otro. Ungerson (1987: 83) estima que las mujeres son «aparentemente las personas apropiadas para combinar el cuidado con el trabajo pagado, el cuidado de los niños y el de las personas dependientes de una generación distinta a la de ellas». 


\section{Conclusiones}

El análisis de los resultados conduce a diversas conclusiones: que la familia continúa siendo el principal sistema de bienestar, pero que las familias - ni las personas- no pueden ser forzadas a ocuparse de un enfermo o una enferma discapacitado; que las relaciones interpersonales son complejas y el conflicto está presente en ellas; que los servicios sociales deben proporcionar soluciones racionales a las personas enfermas y a sus cuidadoras, tanto por medio de la institucionalización del enfermo o de la enferma, como del apoyo exterior a la familia cuidadora; que las personas que deseen cuidar en casa de una persona enferma deben recibir apoyo intra y extrafamiliar en su trabajo. Es muy necesario también un apoyo emocional que haga más Ilevadera la tarea y evite la incidencia negativa del deterioro de la situación, tanto en la persona cuidadora como en la cuidada, pues la relación entre ambas es circular. De las diversas situaciones analizadas, se observa claramente que conforme se asume con mayor voluntariedad el cuidado de una persona anciana discapacitada, los recursos psicológicos propios (percepción del hecho, actitud ante el mismo, estado de ánimo) son mayores y mejores, permitiendo hacer frente a la situación de forma menos dañina.

Al terminar la presente investigación se tiene la sensación de haber penetrado en un mundo desconocido. Las personas cuidadoras, que son principalmente mujeres, se encuentran en España en una situación de abandono por parte de los servicios sociales formales e informales, y también del sistema sanitario, que no les proporciona a veces ni siquiera información. N ormalmente cuentan con el apoyo material y emocional de la familia nuclear: cónyuge e hijos, hijas, y en bastantes casos también con el de la familia extensa: hermanos y hermanas biológicos y políticos, sobre todo. O tros parientes (primos y primas), así como vecinos y vecinas, amigos y amigas proporcionan principalmente compañía y apoyo emocional.

Las personas cuidadoras se sienten abandonadas por parte de los sistemas de salud y de servicios sociales. La mayor parte de ellas ni siquiera ha solicitado ayuda. Son dos principalmente las razones: entienden que los servicios sociales formales son escasos, y los privados cuando existen, caros, y en la mayor parte de los casos no pueden hacer frente a ellos. O tra razón importante es la idea de asumir naturalmente el cuidado del familiar enfermo. Es sociológicamente interesante preguntarse por las causas. Las razones expresadas, las causas manifiestas son que se trata del padre, madre, cónyuge, como razón suprema. 0 tras veces, se habla de reciprocidad («él/ella cuidó antes de nosotros»). También se hace referencia a la obligación de la familia de cuidar a sus familiares enfermos. La mayor parte de las veces las razones aducidas se basan en la obligación y el afecto conjuntamente. Raras veces, sin embargo, se habla de amor. Pero el cariño y el afecto se expresan frecuentemente.

Se han observado procesos de negociación: entre el padre y los hijos y/o hijas, o entre hermanos y/o hermanas. Y también procesos de asignación: cuando un miembro de la familia (una mujer) era designada por los otros miembros 
próximos de la familia para ejercer el rol de cuidadora. Pero, como en otras investigaciones (Ungerson, 1987: 57; Walker, 1991b: 106), lo sociológicamente interesante es que en la presente investigación se observa una construcción social del rol de cuidadora, basada en creencias tradicional es acerca de las funciones protectoras de la familia, y sobre todo de las mujeres como las proveedoras naturales de cuidados.

Puede decirse que ésa es la razón latente, no expresada, de por qué cuidan las mujeres, ya que lo hacen sientan o no amor por la persona cuidada, existan o no sentimientos de reciprocidad, hayan sido las relaciones previas buenas o malas, existan posibilidades materiales adecuadas o no (disponibilidad de tiempo, condiciones de la vivienda). Las personas cuidan por una suerte de imperativo social (aunque además se sienta amor por la persona cuidada). Pero esa coerción moral durkheimiana no suel e percibirse, precisamente por como ha sido interiorizada en los procesos de socialización. Y todavía la familia sigue transmitiendo esos val ores a las generaciones jóvenes (Bazo, 1994a). Es por lo que puede predecirse que en el futuro las personas pueden sufrir serios conflictos.

La estructura social en España ha experimentado fuertes cambios a la par que la economía, el mercado de trabajo o los sistemas normativos. La mayor parte de las mujeres cuidadoras de hoy son amas de casa. Las tasas de actividad económica femenina en España son las más bajas de la Unión Europea, pero la población universitaria española representa en el conjunto de la población joven un porcentaje más alto que en muchos otros países económicamente desarrollados. Entre las jóvenes y los jóvenes universitarios las chicas representan al go más del 50\%. Es de esperar que, como ya se observa, las mujeresjóvenes irán incrementando su presencia en el mercado de trabajo, y se producirá un choque entre los valores interiorizados de apoyo a los miembros necesitados de la familia y aquéllos acerca de la necesidad de autorrealización personal, y más hedonistas e individualistas. Además, cada vez resulta más necesario en una familia disponer de dos salarios.

O bviamente, no se pretende defender con eso que las mujeres son las que deberán seguir cuidando. Simplemente, sugerir la posibilidad de que surjan conflictos, y que la principal fuente tradicional de cuidados se debilitará. Se entiende que la crisis principal de los sistemas de bienestar será una crisis en la provisión de cuidados. Todo ello puede forzar la creación en España de servicios social es provistos por organizaciones voluntarias, y aumentar los privados. Puede también incrementarse la demanda de servicios social es públicos, y puede llegarse a un reparto más equitativo entre géneros de las responsabilidades familiares. Lo cierto es que puede predecirse con casi toda seguridad que el estado de la situación no será igual en el futuro, pero también puede pronosticarse que las mujeres seguirán durante mucho tiempo sintiéndose las más responsables, y las más obligadas. Se produce así una paradoja, que para conseguir la inclusión social de una parte de la población - las personas ancianas y dependientes- se produce la exclusión social de otra parte de la población: las personas cuidadoras. Es de esperar que las políticas sociales no sigan refor- 
zando ese papel que les lleva a ocupar posiciones subordinadas, tanto en la esfera pública como en la privada.

\section{Bibliografía}

ABEL, Emily (1989). «T he ambiguities of social support: Adult daughters caring for frail el derly parents». Journal of Aging Studies, 3: 211-230.

ALLAN, Graham (1990). Family Life. O xford: Blackwell.

BAzo, M aría Teresa (1995). «El reto del envejecimiento: una reflexión sociológica».

Revista Española de Geriatría y Gerontología, 30 (2): 95-97.

- (1992a). La ancianidad del futuro. Barcelona: SG.

- (1994a). «La familia como centro privilegiado de intercambio entre generaciones». Premi os Bancaixa, 1993, p. 269-373. Valencia: Bancaixa.

BAzo, M aría Teresa; D o m ín guez-A ccón , Carmen (1996). «Los cuidados familiares de salud en las personas ancianas, y las políticas sociales». Revista Española de Investigaciones Sociológicas, 73: 43-56.

BÉLAN D, François; ZUN ZUN EGUI, M a Victoria (1995). Envejecer en Leganés. N úmero monográfico de Revista de G erontología, vol. 5, núm. 4. (Ver especialmente capítulos 8 y 9.)

BorgotTA, Edgar F.; M ONTGOMERY, Rhonda J.V. (1987). «Aging policy and socie tal values». En BORGOTTA, Edgar F.; M ONTGOMERY, Rhondaj.V. (eds.). Critical Issues in Aging Policy: Linking Research and Values. Londres: Sage Publications, p. 7-22.

EVERS, Addbert; LEICH SENRIN G, Kai (1994). «Asistencia informal remunerada: una cuestión de creciente importancia». Revista de Gerontología, 4, 2: 114-124.

Fraiz Calvo, Xesús (1993). A poboación centenaria de Galicia. Santiago de Compostel·la: Universidade de Santiago de Compostela.

GIBSON, M ary Jo (1992). «Public health and social policy». En KENDING, H al I.; H ASH IM OTO, A.; C O PPARD, L.C. (eds.). Family Support for the Elderly. O xford: O xford University Press, p. 88-114.

H UNTER, D avid; M ACPHERSON, I sabel (1993). «nfluencia de los cuidadores informales sobre la provisión de servicios y las decisiones de asignación». En J AM IESON, Anne; I LLSLEY, Raymond. Comparación de políticas europeas de atención a las personas ancianas. Barcelona: SG .

JAM IESO N, Anne; I LLSLEY, Raymond (1993). Comparación de políticas europeas deatención a las personas ancianas. Barcelona: SG .

LEIBFRIED, Stephan (1993). «Towards a european welfare state?». En JONES, C atherine (ed.). N ew Perspectives on the Welfare State in Europe. Londres: Routledge.

LEWIS, Jane; M EREDITH, Barbara (1988). D aughters who Care: D aughters C aring for M others at H ome. Londres: Routledge, 1988.

M AYo, M arjorie (1994). Communities and Caring: The M ixed Economy of Welfare. Londres: The M acM illan Press.

M IGUEL, Jesús M . de y otros (1994). C apítulo 6 «Salud y sanidad». En Q uinto informe sociológi co sobre la situación social en España: la salud para todos en el año 2000. M adrid: Fundación Foessa, 2 vols.

M INISTERIO de Asuntos So Ciales (1995). Cuidados en la vejez. El apoyo informal. $M$ adrid: M inisterio de Asuntos Sociales. 
M ONTGOM ERY, Rhonda J.V.; H ATCH, LaurieR. y otros (1987). «D ependency, family extension, and long-term care policy». En BORGOTTA, Edgar F.; M ONTGOMERY, R honda J.V. (eds.). C ritical I ssues in Aging Policy: Linking Research and Values. Londres: Sage Publications, p. 161-177.

M YLES, John (1991). «Editorial: Women, the welfare state and caregiving». Canadian Journal on Aging / La Revue canadienne du vieillissement, vol. 10, 2: 82-85.

OCDE (1987). Financing and D elivering $H$ ealth Care. París: O CDE.

O RY, M arcia G .; BON D, K athleen (eds.) (1989). « ntroduction: H ealth care for an aging society». En Aging and $\mathrm{H}$ ealth Care: Social Science and Policy Perspectives. Londres: Routledge, p. 265.

PEACE, Sheila (1991). «The forgotten female: Social policy and older women». En PhILlipson, Chris; W ALKer, Alan (eds.). Ageing and Social Policy: A Critical Assessment. Aldershot: G ower.

Po Licy St udies I N ST ITUTE (1982). Family Care of the H andicapped Elderly: Who Pays? Londres: PSI.

Q URESH I H AZEL; W ALKER, Alan (1991). «C aring for elderly people: the family and the state». En PhILlIPSON, Chris; W ALKer, Alan (eds.). Ageing and Social Policy: A Critical Assessment. Aldershot: G ower, p. 109-127.

Rod ríguez Cabrero, G regorio (1994). «Políticas de rentas». En Q uinto informe sociológico sobre la situación social en España: La salud para todos en el año 2000. M adrid: Fundación Foessa, 2 vols. Capítulo 9.

SÁN CHEZ-O STIZ, Rafael (1997). «A proximación al estado socio-sanitario de los nonagenarios de Pamplona: estado funcional, cognitivo, nutricional, hemático e inmunológico». Revista Española de Geriatría y Gerontología, 32 (3): 183-184.

SCH ULZ, Richard (1990). «T heoretical perspectives on caregiving: concepts, variables, and methods». En D AVID, E.; BLum, Arthur. Aging and Caregiving. Londres: Sage Publications, p. 27-52.

TEN N STEDT; M CK IN LAY (1989). «Informal care for frail older persons». En O RY, M arcia G .; Bon D, K atheleen (eds.). Aging and H ealth Care: Social Science and Policy Perspectives. Londres: Routledge, p. 145-166.

U n GERSO N, Clare (1987). Policy is Personal: Sex, Gender and Informal Care. Londres: Tavistock Publications.

VICTOR, Cristina R. (1991). H ealth and H ealth Care in Later Life. Buckingham: O pen University.

W ALKER, Alan (1984). «T he political economy of privatisation». LE GRAND, J.; ROBENSON, R. (eds.). Privatisation and the Wefare State Londres: Allen and Unwin, p. 38-39.

- (1991a). «Pensions and the production of poverty in old age». En PHILLIPSON , Chris; W ALKER, Alan (eds.). Ageing and Social Policy: A Critical Assessment. Aldershot: Gower.

- (1991b). «T he relationship between the family and the state in the care of older people». Canadian Journal on Aging / La Revue canadienne du vieillissement, vol. 10, 2: 94-112.

W ALKER, Alan; W ARREN, Lorna (1993). «T he care of frail older people in Britain: Current policies and future prospects». En KATZ O LSON, Laura (ed.). The Graying of the World: Who will Care for the Frail Elderly? N ueva York: The H aworth Press. 